\title{
Lo sguardo connettivo: le macchine per disegnare in prospettiva tra XVI e XVII secolo
}

\author{
Laura Farroni \\ Giulia Tarei
}

Abstract

II saggio focalizza l'attenzione sul ruolo connettivo che lo sguardo ha avuto nello sviluppo delle macchine per disegnare tra XVI e XVII secolo. In particolare nei 'prospettografi' l'atto del guardare e del traguardare relaziona l'uomo osservatore con ciò che lo circonda definendo l'evoluzione delle macchine stesse e della Scienza della Rappresentazione. Lo strumento relaziona, di volta in volta, 'problema e soluzione','spazio reale e spazio della rappresentazione', 'tridimensionalità e bidimensionalità'. Emerge che il meccanismo, l'automazione del processo del disegnare attraverso le possibili modalità dello sguardo/traguardo controlla il processo di astrazione in relazione alla realtà materiale. Le rappresentazioni figurative che da questo nesso derivano, incorporano la corrispondenza, quindi, tra modus operandi e modus cogitandi.

Parole chiave

macchine per disegnare, prospettiva, strumenti, ottica, guardare.

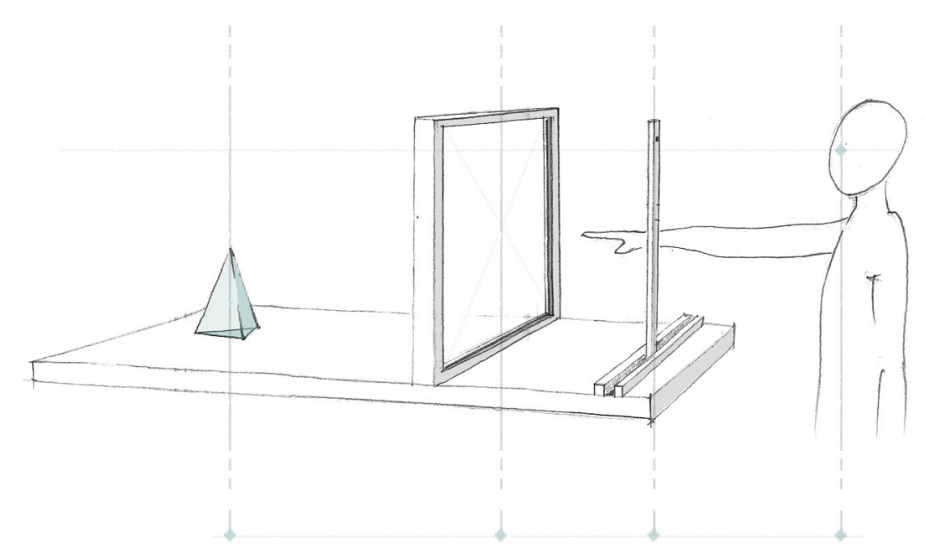




\section{Introduzione}

II tema degli strumenti e metodi per la prospettiva applicata [Salvatore 2020, p. 95] nell'ambito della letteratura scientifica è stato oggetto di numerosi studi in cui si approfondisce l'utilizzo in ambito progettuale, per il riporto di curve e disegni su superfici murarie, o per il rilievo, ma anche gli aspetti relativi ad un uso puramente legato allinterpretazione geometrica dello spazio, o ad uso didattico. La spiegazione del funzionamento delle macchine è, spesso, interpretata attraverso la ricostruzione di modelli, sia fisica che virtuale. Sono, inoltre, molte le collezioni di strumenti conservate all'interno di musei o di sedi universitarie: si segnala a titolo esemplificativo il laboratorio di macchine matematiche dell'Università degli studi di Modena e Reggio Emilia (figg. I, 2).

Molti sono anche i contributi di carattere teorico e le sperimentazioni di autori quali Fabrizio Gay, Filippo Camerota, Andrea Casale, Maria G. Bartolini Bussi, Laura Farroni e Marta Salvatore, le cui indagini hanno talvolta un carattere classificatorio, con una visione ampia che tende ad una sistematizzazione, talvolta si incentrano su un particolare strumento o autore, anche in riferimento alla produzione trattatistica prodotta tra il XVI ed il XVII secolo ormai facilmente consultabile nelle risorse online degli archivi.

Negli ultimi anni, è noto, sono stati resi accessibili molti testi antichi attraverso le digitalizzazioni e la messa in rete in open source.

Con il contributo presentato si intende focalizzare l'attenzione sul ruolo connettivo che lo sguardo ha avuto nello sviluppo delle macchine per disegnare nel periodo in esame. In particolare, nei 'prospettografi' l'atto del guardare e del traguardare relaziona l'uomo osservatore con ciò che lo circonda definendo il funzionamento delle macchine stesse, il modo di descrivere la forma e l'evoluzione della Scienza della Rappresentazione.

Tra il XVI ed il XVII secolo architetti, matematici, ingegneri, astronomi, usano gli strumenti e li adattano a seconda del loro impiego.

Protagonisti del secolo della rivoluzione scientifica sono figure di scienziati che coadiuvarono lo sviluppo di teorie e tecniche per l'analisi e la codifica delle leggi che regolano il mondo fisico. II lavoro e le ricerche che tali personalità portarono avanti nell'ambito della sperimentazione strumentale per la Scienza della Rappresentazione testimonia un aspetto cardine della modalità operativa intrinseca a tale scienza, l'unione tra 'sapere e saper fare'.

Lo strumento si pone dunque come elemento di connessione tra 'problema e soluzione', tra 'spazio reale e spazio della rappresentazione', 'tra tridimensionalità e bidimensionalità'.

Partendo dall'analisi del modo di fissare lo sguardo per ritrarre la realtà, la ricerca si è sviluppata nel comprendere le componenti di alcuni esemplari e le variazioni in ragione del loro utilizzo. I risultati sono stati raccolti in un abaco che presenta una classificazione di strumenti che considera due aspetti: gli elementi che compongono ciascuna macchina e i modi d'uso. Le componenti materializzano gli elementi teorici, il loro assemblaggio garantisce i principi geometrici e il meccanismo replica le operazioni caratterizzanti la rappresentazione prospettica. In essi, quindi, sono riconoscibili: l'osservatore, il raggio visivo, il piano di quadro, e
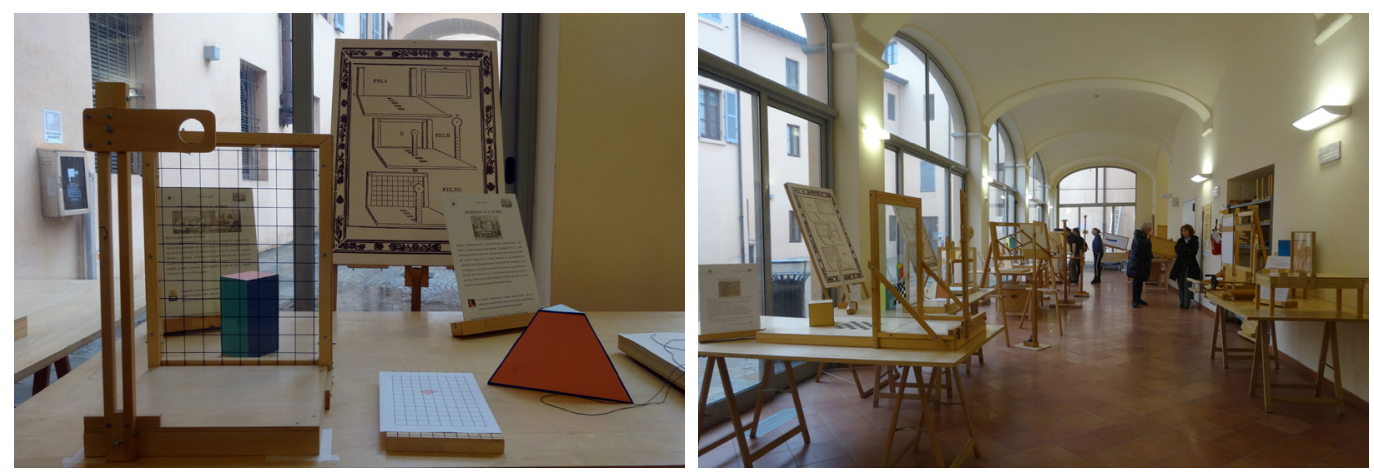
Fig. 3. Vetro di Albrecht Dürer [1525, p. 182].

Fig. 4. Sportello di Albrecht Dürer [1525, p. 183].
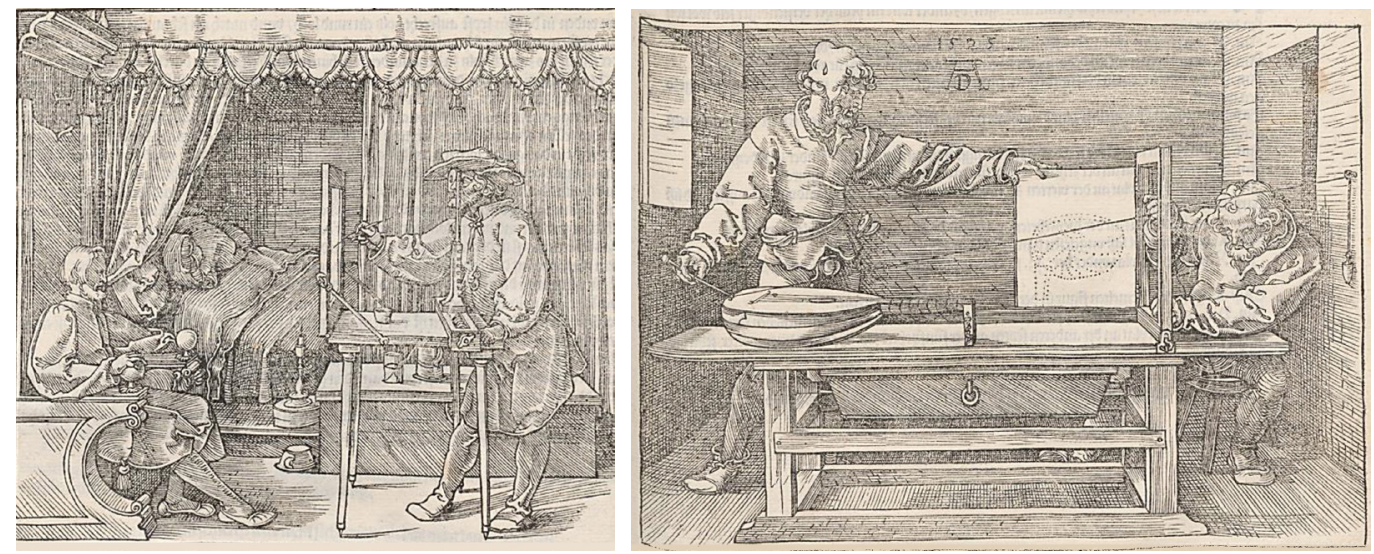

le operazioni di proiezione e sezione. I diversi modi d'uso esplicitano il carattere descrittivo ad essi relativo.

Premessa da fare per discernere e classificare le modalità d'uso è che gli strumenti prospettici inizialmente incorporano in modo diretto la definizione di pittura di Alberti data nel De Pictura, come intersegazione della piramide visiva.

"Quae res cum ita sit, pictam superficiem intuentes intercisionem quandam pyramidis videre videntur. Erit ergo pictura intercisio pyramidis visivae secundum datum intervallum posito centro statutisque luminibus in datam superficiem lineis et coloribus arte repraesentata" [Alberti, 1435].

"Qual cosa se così è quanto dissi, adunque chi mira una pittura vede certa intersegazione d'una piramide. Sarà adunque pittura non altro che intersegazione della piramide visiva, sicondo data distanza, posto il centro e constituiti i lumi, in una certa superficie con linee e colori artificiose representata" [Grayson 1980, p. 7]. La base della piramide è descritta come una finestra (il vetro), su cui si disegna per trasparenza l'immagine dell'oggetto (l'oggetto degradato), e che incorpora in modo diretto l'idea di 'intersegazione'.

\section{Definizione delle metodologie adottate nella ricerca e abaco critico delle macchine prospettiche}

Lo studio si è awvalso delle seguenti metodologie: a. la ricerca storica; b. l'analisi dei principi geometrici e dei procedimenti di alcune macchine esemplificative; c. il progetto e la costruzione di prototipi; $d$. la catalogazione dei dati raccolti in un abaco.

La ricerca si è articolata principalmente secondo le seguenti fasi: sono state analizzate fonti; sulla base delle fonti sono stati realizzati prototipi; dall'analisi dei prototipi e delle fonti si sono ricavati dati per la redazione della tabella che consente una comparazione analitica tra gli strumenti di diversi autori e tra strumenti simili di uno stesso autore. La macchina è la descrizione dei principi a cui i diversi autori danno forma nel realizzare la scena prospettica, che derivano da scelte consapevoli sulla base di esperimenti. Questo aspetto mette in evidenza il carattere empirico del metodo scientifico.

Le macchine prospettiche hanno la proprietà di essere utilizzate con il duplice scopo di: a. acquisizione delle informazioni dimensionali, geometriche e topologiche del mondo fisico identificabile con le operazioni di rilievo; b. operazioni di riporto ossia il trasferimento di un disegno bidimensionale su un supporto fisico tridimensionale curvo o piano.

Nel tentativo di organizzare in maniera sistematica il palinsesto di macchine per il disegno in prospettiva, progettate e realizzate in un arco temporale di due secoli, si sono rintracciati quelli che sono i principi geometrici che le accomunano, e si sono interpretate le varie declinazioni formali che derivano dal progressivo superamento di limiti fisici, o dalle necessità legate ad operazioni specifiche per cui tali strumenti erano progettati. 


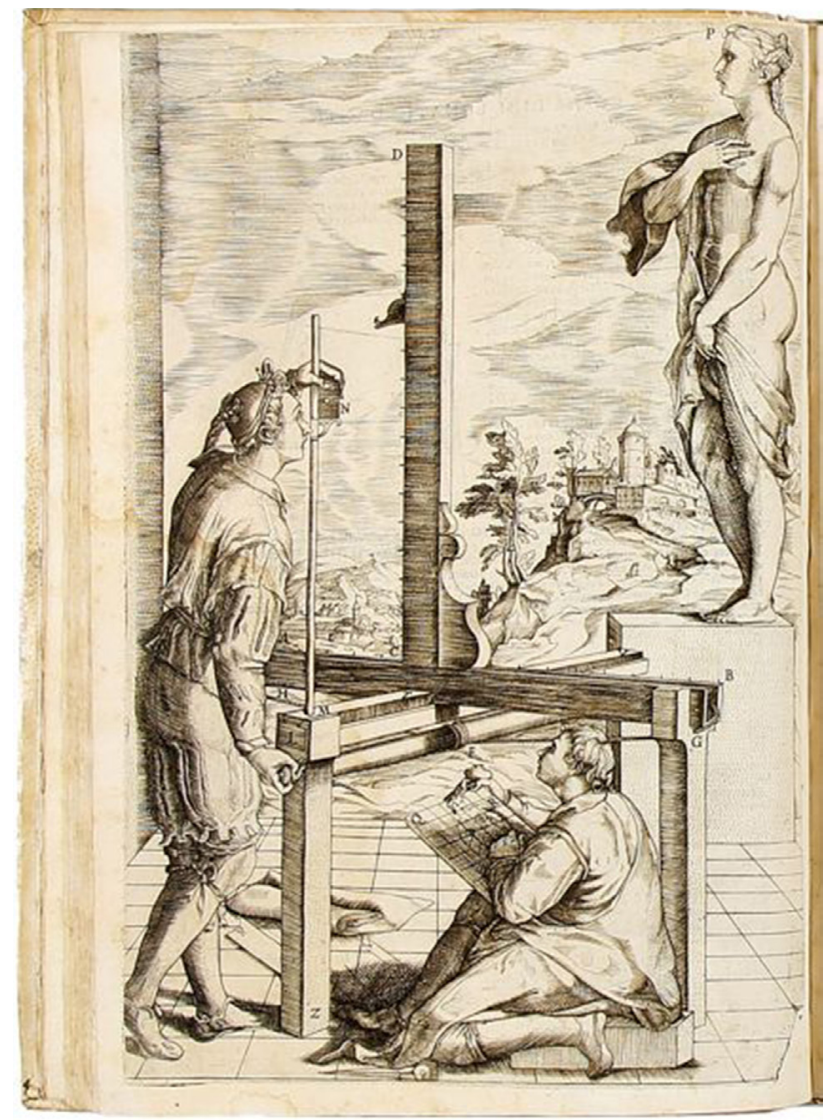

Le macchine analizzate sono:

- Vetro di Albrecht Dürer XVI secolo (fig. 3).

- Griglia di Albrecht Dürer XVI secolo.

- Sportello di Albrecht Dürer XVI secolo (fig. 4).

- Traguardi di Jacob Keser XVI secolo.

- Sportello di Albrecht Dürer con diottra (Egnazio. Danti) XVI secolo.

- Prospettografo dell'abate di Lerino (Don Girolamo da Perugia) XVI secolo.

- Sportello di Egnazio Danti con regoli graduati XVI secolo (fig. 5).

- Prospettografo di Tigini dè Marij XVI secolo.

- Prospettografo di lacomo Barozzi da Vignola XVI secolo.

- Prospettografo di Baldassarre Lanci XVI secolo.

- Prospettografo di Wenzel Jämnitzer XVII secolo.

- Prospettografo di Chrostoph Scheiner XVII secolo (fig. 6).

- Prospettografo di Bettini - Grienberger XVII secolo (fig. 7).

- Prospettografo di Cigoli - Niçeron XVII secolo (fig. 8).

I principi geometrici comuni alle macchine sono quelli che regolano la sequenza operativa di proiezione da un centro proprio e sezione su di un piano, il quale, nella maggior parte dei congegni è orientato verticalmente, ma che può avere orientamento generico purché risulti ortogonale al raggio visivo. I raggi proiettanti con cui si traguarda l'oggetto reale determinano i punti di intersezione sul piano. Le varie declinazioni formali degli strumenti sono legate a questi elementi, alla loro concezione come elementi geometrici ed alla loro traduzione in elementi fisici. Un punto, il centro proprio, le rette proiettanti ed il piano della rappresentazione sono gli enti geometrici che vengono formalmente definiti nelle macchine, sperimentando di volta in volta le combinazioni possibili tra tangibile ed intangibile. 
Fig. 6. Prototipo del 'prospettografo' di Chrostoph Scheiner $\mathrm{XVII}$ sec. realizzato da Marta Pagliaccia nell'ambito del corso di Macchine matematiche. Titolari L. Farroni e P. Magrone. Dipartimento degli Studi di Roma Tre.
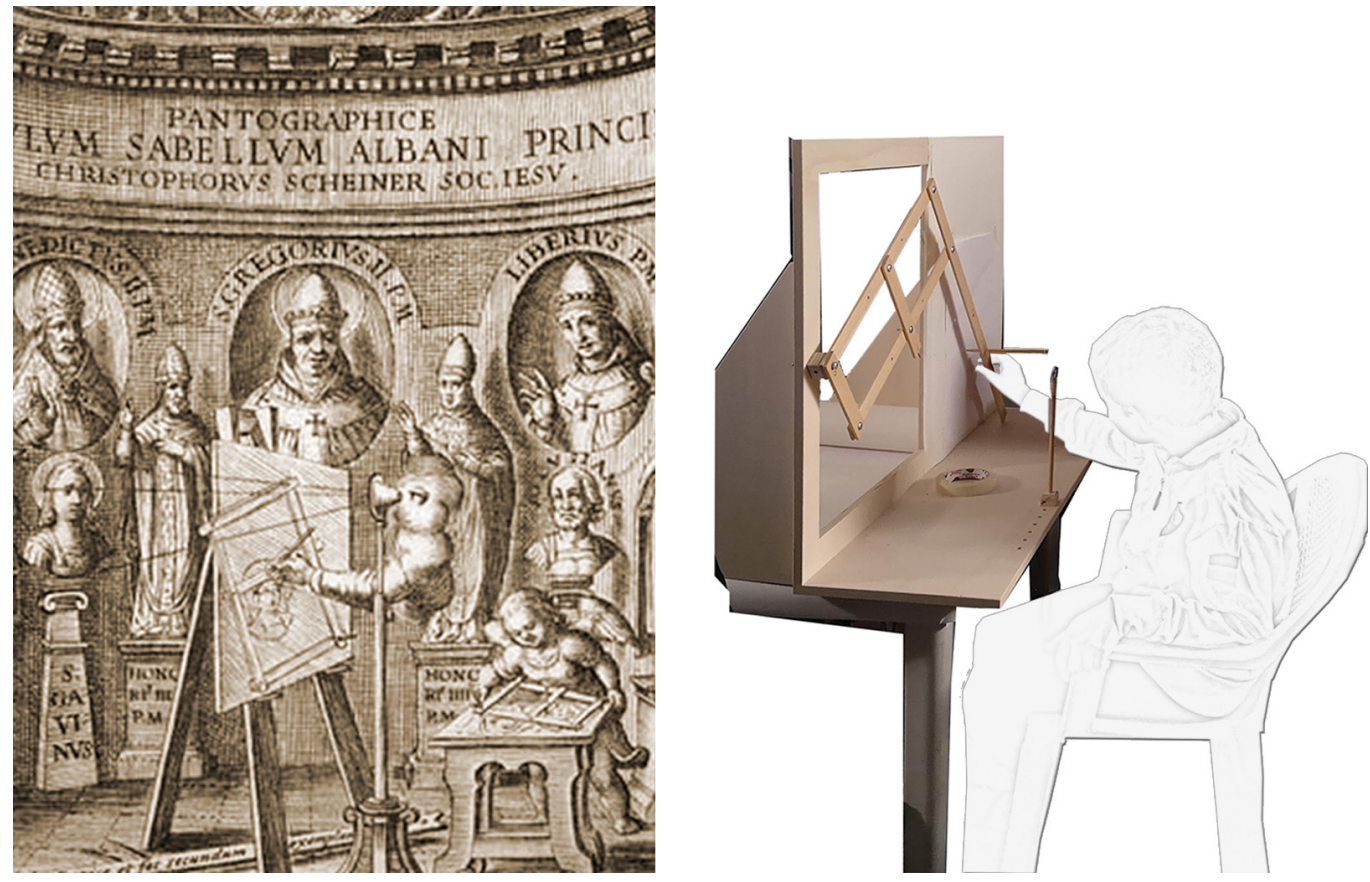

Diverse sono poi le modalità d'uso degli strumenti presi in considerazione. L'utilizzo dello strumento dipende dal sistema assiomatico scelto per una descrizione efficace. Per garantire questa efficacia, è necessario rendere l'operazione 'proiettivo-descrittiva' agevole e rapida, lavorando sulla compattezza e sulla praticità nel progetto della macchina.

Ed ecco appunto che emerge l'importanza dello sguardo (figg. 9- I I). Alcune soluzioni prevedono che il disegnatore resti fermo nella sua posizione di osservatore, con altre - introducendo talvolta elementi ausiliari - si svincola il disegnatore dalla posizione dell'effettivo punto di vista, che resta invariata: il disegnatore può muoversi per riportare il disegno, su supporto integrato alla macchina o a parte, senza modificare l'impostazione prospettica. Da ciò deriva l'escogitare la materializzazione dei raggi visivi per mezzo di fili nello spazio o, attraverso quella che si può definire come una sintesi sul piano di quadro, con squadre e regoli che scorrono su di esso.

Tutto ciò si traduce anche in un'altra caratteristica operativa: la simultaneità delle operazioni di osservazione e disegno, che possono altrimenti avvenire in due fasi distinte, intervallate da una opportuna misurazione. II metodo di trasporto dal piano virtuale al piano del foglio, quando è necessario, chiama in gioco, se pure in modo implicito, trasformazioni geometriche quali, ad esempio, l'omotetia e la traslazione [Bartolini 2006, p. 39].
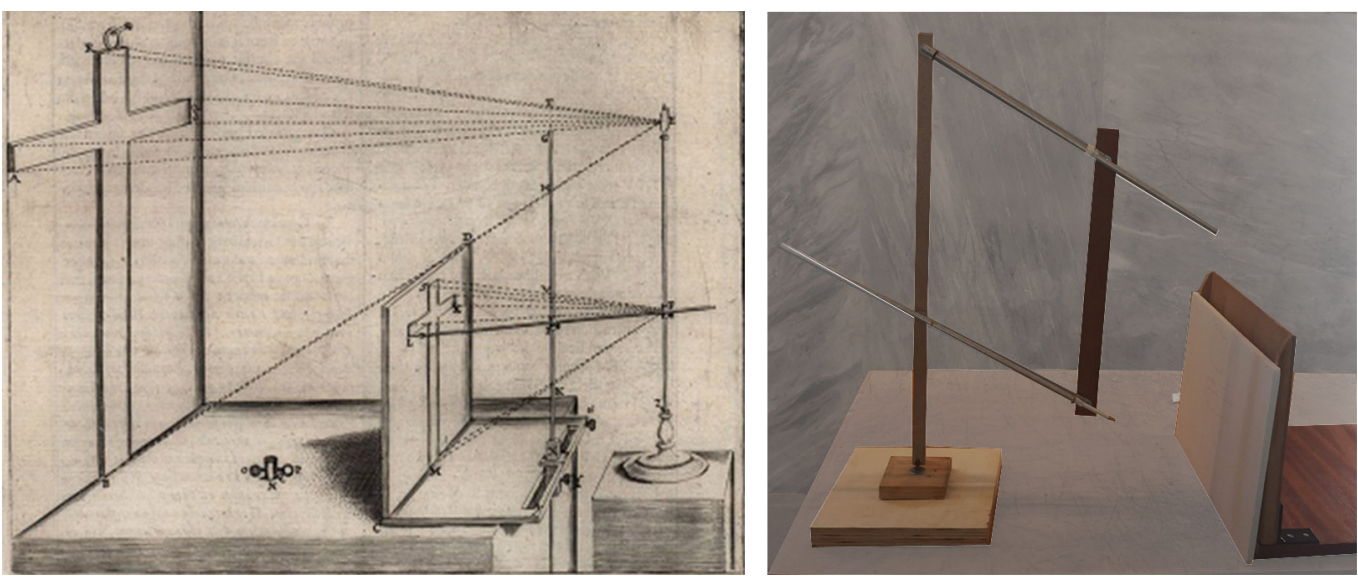

Fig. 7. Prototipo del 'prospettografo' di Bettini realizzato da Chiara Egid nell'ambito del corso di nell ambito del corso Titolari L Farroni P. Magrone. Dipartimento di Architettura. Universita degli Studi di Roma Tre. 
Fig. 8. Prototipo del 'prospettografo' di Cigoli - Niçeron XVII sec realizzato da Giulia Tarei nell'ambito del corso di Macchine matematiche. Titolari L. Farroni e P. Magrone. Dipartimento di Architettura. Università degli Studi di Roma Tre.
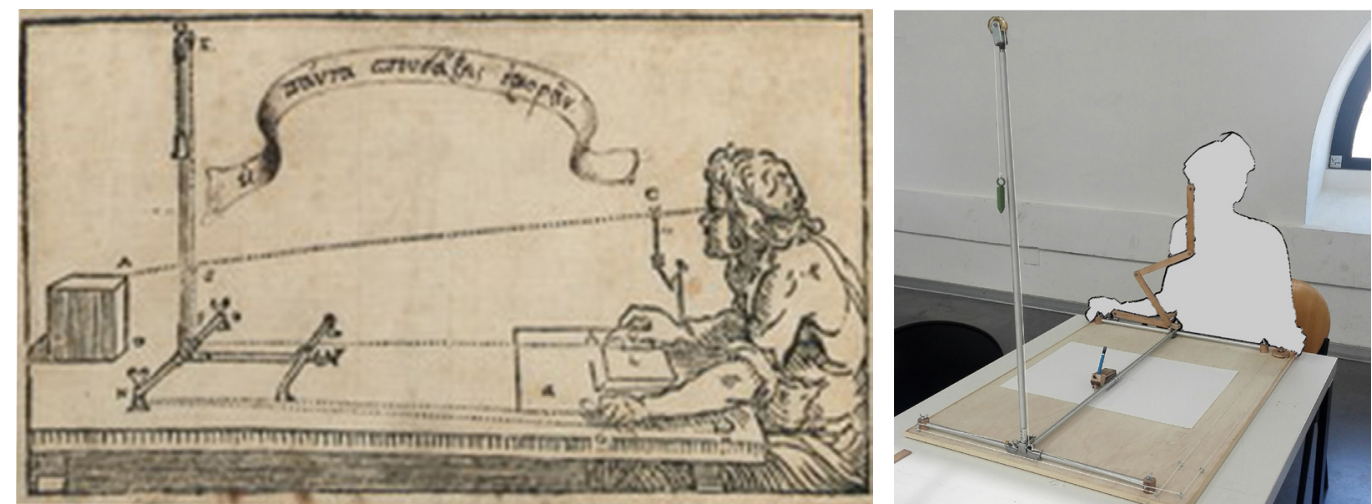

La progettazione dimensionale delle parti componenti e delle reciproche distanze è di tipo antropometrico, al fine di controllarne il funzionamento.

Nelle macchine in esame sono stati rilevati gli espedienti tecnici che relazionano ad esempio la distanza massima del vetro alla lunghezza del braccio dell'osservatore o, ove presente, alla posizione dell'oculare che deve essere accessibile al pittore che guarda dal foro. La materializzazione dei raggi visivi consente di superare il limite dell'oculare: se un raggio è rappresentato da un filo teso, basta un occhiello attraverso cui far passare i fili per simulare un oculare in una posizione qualsiasi.

L'osservatore umano definisce l'unità di misura, ma al tempo stesso, attraverso lo strumento, determina lo spazio da rappresentare, anche superando con strumenti accessori la limitazione antropometrica.

\begin{tabular}{|c|c|c|c|c|c|}
\hline & \multicolumn{5}{|c|}{$\mathrm{XVI}$ secolo } \\
\hline & $\begin{array}{l}\text { vetro } \\
\text { A.Dürer }\end{array}$ & $\begin{array}{l}\text { griglia } \\
\text { A. Dürer }\end{array}$ & $\begin{array}{l}\text { sportello } \\
\text { A. Dürer }\end{array}$ & $\begin{array}{l}\text { traguardo } \\
\text { J. Keser }\end{array}$ & $\begin{array}{l}\text { sportello } \\
\text { A. Dürer / E. Danti }\end{array}$ \\
\hline osservatore & $\begin{array}{l}\text { conicidente con } \\
\text { punto di vista }\end{array}$ & $\begin{array}{l}\text { conicidente con } \\
\text { punto di vista }\end{array}$ & $\begin{array}{l}\text { non coincidente } \\
\text { con punto di vista }\end{array}$ & $\begin{array}{l}\text { non coincidente } \\
\text { con punto di vista }\end{array}$ & $\begin{array}{l}\text { conicidente con punto } \\
\text { di vista }\end{array}$ \\
\hline punto di vista & oculare & oculare & chiodo su parete & chiodo su parete & diottra \\
\hline piano di quadro & $\begin{array}{l}\text { telaio verticale } \\
\text { con vetro }\end{array}$ & $\begin{array}{l}\text { telaio verticale } \\
\text { con griglia }\end{array}$ & $\begin{array}{c}\text { telaio verticale } \\
\text { con fili diagonali }\end{array}$ & $\begin{array}{l}\text { telaio verticale } \\
\text { con vetro }\end{array}$ & $\begin{array}{l}\text { telaio verticale con fili } \\
\text { diagonali e sportello } \\
\text { con foglio da disegno }\end{array}$ \\
\hline piano orizzontale & piano di supporto & $\begin{array}{l}\text { foglio da disegno } \\
\text { con griglia }\end{array}$ & piano di supporto & piano di supporto & piano di supporto \\
\hline raggio visivo & immateriale & immateriale & filo & filo & filo \\
\hline $\begin{array}{l}\text { elemento } \\
\text { ausiliario }\end{array}$ & - & - & $\begin{array}{c}\text { sportello con } \\
\text { foglio da disegno }\end{array}$ & regolo - visore & $\begin{array}{l}\text { diottra } \\
\text { sportello }\end{array}$ \\
\hline disegnatore & osservatore & osservatore & osservatore & $\begin{array}{l}\text { non coincidente } \\
\text { con l'osservatore }\end{array}$ & osservatore \\
\hline operatori & 1 & 1 & 2 & 2 & 2 \\
\hline scopo & rilievo & rilievo & rilievo & rilievo & rilievo \\
\hline modo d'uso & diretto & indiretto & indiretto & diretto & indiretto \\
\hline operazioni & ricalco sul vetro & $\begin{array}{l}\text { 1.individuazione } \\
\text { di punti su griglia } \\
\text { verticale } \\
\text { 2.riporto su foglio } \\
\text { con griglia }\end{array}$ & $\begin{array}{l}\text { 1.individuazione } \\
\text { di punti su telaio } \\
\text { con diagonali } \\
\text { 2.riporto su } \\
\text { sportello con } \\
\text { foglio }\end{array}$ & disegno sul vetro & $\begin{array}{l}\text { 1.individuazione di } \\
\text { punti su telaio con } \\
\text { diagonali 2.riporto } \\
\text { su sportello con foglio }\end{array}$ \\
\hline $\begin{array}{l}\text { limite } \\
\text { antropometrico }\end{array}$ & $\begin{array}{l}\text { lunghezza del } \\
\text { braccio }\end{array}$ & - & - & - & - \\
\hline limite operativo & $\begin{array}{l}\text { disegnatore/ } \\
\text { osservatore in } \\
\text { posizione } \\
\text { vincolata }\end{array}$ & $\begin{array}{l}\text { disegnatore/ } \\
\text { osservatore in } \\
\text { posizione } \\
\text { vincolata }\end{array}$ & $\begin{array}{l}\text { necessità di un } \\
\text { assistente e di } \\
\text { una parete }\end{array}$ & $\begin{array}{l}\text { necessità di un } \\
\text { assistente e di } \\
\text { una parete }\end{array}$ & $\begin{array}{l}\text { necessità di un } \\
\text { assistente }\end{array}$ \\
\hline $\begin{array}{l}\text { limite di } \\
\text { precisione }\end{array}$ & - & $\begin{array}{l}\text { errori dovuti al } \\
\text { riporto su griglia }\end{array}$ & $\begin{array}{l}\text { errori dovuti allo } \\
\text { spostamento del } \\
\text { filo }\end{array}$ & - & $\begin{array}{l}\text { errori dovuti allo } \\
\text { spostamento del filo }\end{array}$ \\
\hline
\end{tabular}




\begin{tabular}{|c|c|c|c|c|c|}
\hline & \multicolumn{5}{|c|}{ XVI secolo } \\
\hline & $\begin{array}{l}\text { prospettografo } \\
\text { Abate da Lerino }\end{array}$ & $\begin{array}{c}\text { prospettografo } \\
\text { E. Danti }\end{array}$ & $\begin{array}{l}\text { prospettografo } \\
\text { O. Trigini De' Marij }\end{array}$ & $\begin{array}{l}\text { prospettografo } \\
\text { I. Barozzi da Vignola }\end{array}$ & $\begin{array}{c}\text { prospettografo } \\
\text { B. Lanci }\end{array}$ \\
\hline osservatore & $\begin{array}{l}\text { conicidente con } \\
\text { punto di vista }\end{array}$ & $\begin{array}{l}\text { conicidente con } \\
\text { punto di vista }\end{array}$ & $\begin{array}{l}\text { conicidente con } \\
\text { punto di vista }\end{array}$ & $\begin{array}{l}\text { conicidente con } \\
\text { punto di vista }\end{array}$ & $\begin{array}{l}\text { conicidente con punto } \\
\text { di vista }\end{array}$ \\
\hline punto di vista & oculare & oculare & osservatore & oculare & oculare \\
\hline piano di quadro & $\begin{array}{l}\text { telaio verticale e } \\
\text { sportello con foglio } \\
\text { da disegno }\end{array}$ & $\begin{array}{c}\text { sistema di aste } \\
\text { graduate } \\
\perp \text { tra di loro }\end{array}$ & $\begin{array}{l}\text { telaio per } \\
\text { osservare }\end{array}$ & $\begin{array}{l}\text { sistema di aste } \\
\text { graduate } \\
\perp \text { tra di loro }\end{array}$ & piano cilindrico \\
\hline piano orizzontale & piano di supporto & foglio da disegno & - & asta orizzontale & piano di supporto \\
\hline raggio visivo & immateriale & immateriale & immateriale & immateriale & immateriale \\
\hline $\begin{array}{l}\text { elemento } \\
\text { ausiliario }\end{array}$ & $\begin{array}{l}\text { squadre - righelli } \\
\text { su piano di quadro }\end{array}$ & $\begin{array}{l}\text { due regoli graduati } \\
\perp \text { tra di loro }\end{array}$ & $\begin{array}{l}\text { - foglio da disegno } \\
\text { contiguo al telaio } \\
\text { - filo }\end{array}$ & $\begin{array}{c}\text { regoli } \\
\text { foglio con griglia }\end{array}$ & puntatore \\
\hline disegnatore & osservatore & osservatore & osservatore & assistente & osservatore, puntatore \\
\hline operatori & 1 & 1 & 1 & 2 & 1 \\
\hline scopo & rilievo & rilievo e riporto & rilievo & rilievo e riporto & rilievo e riporto \\
\hline modo d'uso & indiretto & indiretto & diretto & indiretto & diretto \\
\hline operazioni & $\begin{array}{l}\text { 1.individuazione di } \\
\text { punti su telaio con } \\
\text { righelli 2.riporto su } \\
\text { sportello con foglio }\end{array}$ & $\begin{array}{l}\text { 1.individuazione } \\
\text { di punti con regolo } \\
\text { verticale } \\
\text { 2.riporto su foglio } \\
\text { con regolo } \\
\text { orizzontale }\end{array}$ & $\begin{array}{l}\text { osservazione su } \\
\text { quadro virtuale e } \\
\text { riporto su quadro } \\
\text { reale prendendo le } \\
\text { misure su un } \\
\text { regolo }\end{array}$ & $\begin{array}{l}\text { 1.individuazione } \\
\text { di punti su aste } \\
\text { graduate } \\
\text { 2.riporto di misure } \\
\text { su foglio con } \\
\text { griglia }\end{array}$ & $\begin{array}{l}\text { operazioni simultanee } \\
\text { di osservazione e } \\
\text { disegno }\end{array}$ \\
\hline $\begin{array}{l}\text { limite } \\
\text { antropometrico }\end{array}$ & - & - & - & - & - \\
\hline limite operativo & - & - & - & $\begin{array}{l}\text { - complessità } \\
\text { dello strumento - } \\
\text { necessità di un } \\
\text { assistente }\end{array}$ & $\begin{array}{l}\text { lo strumento consente } \\
\text { di riportare un disegno } \\
\text { in prospettiva su } \\
\text { parete curva }\end{array}$ \\
\hline $\begin{array}{l}\text { limite di } \\
\text { precisione }\end{array}$ & - & - & - & - & - \\
\hline
\end{tabular}

\section{Conclusioni}

Questo primo lavoro, in cui sono messe a confronto le caratteristiche delle macchine analizzate, presenta un quadro evolutivo critico del processo tecnologico. Gli strumenti funzionano come modelli geometrici, espressione di un certo aspetto o fenomeno: materializzano un costrutto immateriale di idee, connettendo il sistema astratto di segni codificati che li possono descrivere ad un artefatto tangibile [Seidl 20I 8, p. 20].

Dallo studio e l'analisi delle fonti bibliografiche, sulla base delle descrizioni degli autori, è derivata la realizzazione di prototipi. Dal mettere in relazione gli elementi dell'indagine, ossia le fonti ed i prototipi, si sono focalizzati gli aspetti comuni e le proprietà geometriche, nonché i dati con cui strutturare la tabella. Vengono messe in evidenza anche le connessioni logiche, cronologiche e le interazioni tra i vari scienziati. Nella comparazione tra i prototipi analizzati, l'uso di fili per simulare il raggio visivo accomuna, ad esempio, lo sportello di Dürer al traguardo di Keser, allo strumento di Jämnitzer realizzato un secolo dopo. $O$ ancora, i vari tentativi di svincolare il disegnatore dalla posizione dell'osservatore - in particolare per le operazioni di rilievo e riporto - trovano esito nella presenza di due operatori ad esempio nelle sperimentazioni più mature di Dürer, o in quelle di Danti, di Keser o del Vignola, prima di giungere alle automazioni consentite dagli strumenti di Bettini o del Cigoli. Emerge che II meccanismo, l'automazione del processo connette 


\begin{tabular}{|c|c|c|c|c|}
\hline & \multicolumn{4}{|c|}{ XVII secolo } \\
\hline & $\begin{array}{c}\text { prospettografo } \\
\text { W. Jämnitzer }\end{array}$ & $\begin{array}{c}\text { prospettografo } \\
\text { C. Scheiner }\end{array}$ & $\begin{array}{l}\text { prospettografo } \\
\text { Bettini - Grienberger }\end{array}$ & $\begin{array}{l}\text { prospettografo } \\
\text { L.Cigoli - J.F. Niceron }\end{array}$ \\
\hline osservatore & $\begin{array}{l}\text { conicidente con } \\
\text { punto di vista }\end{array}$ & conicidente con punto di vista & $\begin{array}{l}\text { conicidente con } \\
\text { punto di vista }\end{array}$ & $\begin{array}{c}\text { conicidente con punto } \\
\text { di vista }\end{array}$ \\
\hline punto di vista & oculare & oculare & oculare & oculare \\
\hline piano di quadro & $\begin{array}{l}\text { sistema di aste } \\
\perp \text { tra di loro }\end{array}$ & telaio per osservare & foglio verticale & $\begin{array}{l}\text { sistema di aste } \\
\text { mobili } \perp \text { tra di loro }\end{array}$ \\
\hline piano orizzontale & foglio da disegno & - & - & $\begin{array}{l}\text { piano di supporto con } \\
\text { foglio da disegno }\end{array}$ \\
\hline raggio visivo & filo & immateriale & $\begin{array}{l}\text { asta orizzontale } \\
\text { (radius visualis) }\end{array}$ & immateriale \\
\hline $\begin{array}{l}\text { elemento } \\
\text { ausiliario }\end{array}$ & $\begin{array}{l}\text { asta verticale } \\
\text { graduata } \\
\text { pianta e alzato }\end{array}$ & $\begin{array}{l}\text { - foglio da disegno contiguo } \\
\text { al telaio } \\
\text { - parallelogramma articolato } \\
\text { - puntatore e tracciatore }\end{array}$ & $\begin{array}{l}\text { asta orizzontale } \\
\text { (radius scriptorius) } \\
\text { perno per far ruotare } \\
\text { l'asta verticale }\end{array}$ & carrucole e cursore \\
\hline disegnatore & osservatore & osservatore & osservatore & osservatore \\
\hline operatori & 1 & 1 & 1 & 1 \\
\hline scopo & rilievo e riporto & rilievo & rilievo e riporto & rilievo e riporto \\
\hline modo d'uso & indiretto & diretto & diretto & diretto \\
\hline operazioni & $\begin{array}{l}\text { 1.individuazione } \\
\text { di punti in pianta } \\
\text { e alzato } \\
\text { 2.riporto di quote } \\
\text { su asta verticale } \\
\text { 3.ribaltamento } \\
\text { asta e misure su } \\
\text { piano orizzontale }\end{array}$ & $\begin{array}{l}\text { - operazioni simultanee di } \\
\text { osservazione e disegno } \\
\text { - lo strumento sfrutta la } \\
\text { trasformazione geometrica } \\
\text { omotetica } \\
\text { - automatismo }\end{array}$ & $\begin{array}{l}\text { - operazioni } \\
\text { simultanee di } \\
\text { osservazione e } \\
\text { disegno } \\
\text { - lo strumento sfrutta } \\
\text { la trasformazione } \\
\text { geometrica di } \\
\text { traslazione } \\
\text { - automatismo }\end{array}$ & $\begin{array}{l}\text { - operazioni } \\
\text { simultanee di } \\
\text { osservazione e } \\
\text { disegno } \\
\text { - automatismo }\end{array}$ \\
\hline $\begin{array}{l}\text { limite } \\
\text { antropometrico }\end{array}$ & - & - & - & - \\
\hline limite operativo & $\begin{array}{l}\text { lo strumento } \\
\text { prevede l'utilizzo } \\
\text { di disegni ausiliari } \\
\text { pianta e alzato }\end{array}$ & - & - & - \\
\hline $\begin{array}{l}\text { limite di } \\
\text { precisione }\end{array}$ & - & - & - & - \\
\hline
\end{tabular}

anche la più elevata astrazione con la realtà materiale e le rappresentazioni figurative che da questo nesso derivano sono il risultato della corrispondenza tra modus operandi e modus cogitandi.

È proprio la possibilità di descrivere un particolare procedimento per rilevare o disegnare attraverso una macchina a legarsi storicamente alla scelta di un sistema assiomatico e questo può indicare lo stato di avanzamento delle conoscenze proiettive e geometriche oltre ad essere un aspetto che determina la varietà di soluzioni strumentali adottate per riprodurre il disegno in prospettiva. La proiezione dei punti dell'oggetto fisico e l'intersezione tra raggi proiettanti e piano della rappresentazione vengono infatti interpretati e resi corporei con diverse soluzioni nella progettazione dei vari strumenti, e ad ogni scelta non solo corrisponde una determinata necessità pratica e un particolare risultato figurativo, ma un controllo della descrizione geometrica delle curve rappresentate.

L'obiettivo della redazione dell'abaco è stato quello di definire l'evoluzione dei saperi tecnici, legati allo sviluppo teorico.

Ogni macchina e quindi ogni parametro che contiene è frutto di una scelta consapevole in base alle conoscenze; ad ogni strumento corrisponde una selezione delle informazioni da incorporare in esso, una sintesi, quindi, degli aspetti teorici essenziali.

Le autrici procederanno ad evidenziare la gestione dei parametri significativi per veicolare l'approccio parametrico, per un possibile trasferimento nell'uso di applicazioni digitali. Per questo le strade della ricerca sono ancora aperte. 


\section{Riferimenti bibliografici}

Barbaro D. (I568). La pratica della perspettiva ... Opera molto vtile a pittori, a scultori, \& ad architetti. Venezia: Camillo, \& Rutilio Borgominieri fratelli al segno di S. Giorgio.

Bartoli M.T., Lusoli M. (20 15). Le teorie, le tecniche, i repertori figurativi nella prospettiva d'architettura tra il '400 e il '700. Firenze: University Press.

Bartolini Bussi M.G., Maschietto M. (2006). Macchine matematiche: dalla storia alla scuola.Verlag: Springer.

Bettini M. ( 645$)$. Apiaria Universae Philosophiae Mathematicae. Bologna: JO. Bapt. Ferronius - Bononiae.

Camerota F. (2003). Il distanziometro di Baldassarre Lanci: prospettiva e cartografia militare alla corte dei Medici. In Galluzzi P. Beretta M.,Triarico C. (a cura di). Musa Musaei. Studies on Scientific Instruments and Collections in Honour of Mara Miniati. Firenze: Leo S. Olschki Editore, pp. 79-92

Camerota F. (20 I0). Arte e Scienza da Leonardo a Galileo. Firenze: Giunti.

Camerota F. (20 I0). Linear Perspective in the Age of Galileo. Ludovico Cigoli's Prospettiva pratica. Firenze: Leo S. Olschki Editore.

Casale A. (20। 8). Forme della percezione. Dal pensiero all'immagine. Milano: FrancoAngeli.

Cigoli Cardi L. ( | 6 | 3). II Cigoli. Prospettiva pratica di Fra Lodovico Cardi Cigoli Cavaliere della Sacra et Illustre Religione di San Giovanni Hierosolomitasano, dimostrata con tre regole e la descrizione di due strumenti da tirare in prospettiva e modo di adoperarli, et cinque ordini di architettura con le loro misure. Firenze: Gabinetto Disegni e Stampe degli Uffizi.

Danti E. (I583). Le due regole della prospetiva pratica di M.lacomo Barozzi da Vignola. con commentarii del reverendo Padre Maestro Egnatio Danti dell'ordine dei predicatori matematico dello studio di Bologna. Roma: Francesco Zannetti.

Dürer A. ( 1525). Underweysung der Messung mit dem Zyrckel und Richtscheyt. Nurnberg: Hieronymus Andreae.

Farroni L., Magrone P. (20 I 8). A Hands-on Laboratory with Mathematical Mechanical Drawing Machines. In Conference Proceedings, Bridges 2018 Mathematics, Art, Music, Architecture, Education, Culture. Stoccolma 25-27 luglio 20 I 8, pp. 61 7-622.

Farroni L., Magrone P. (2019). A Multidisciplinary Approach to Teaching Mathematics and Architectural Representation: Historical Drawing Machines. Relations between Mathematics and Drawing. In History and Pedagogy of Mathematics, July 2016 , Montpellier, France. <https://hal.archives-ouvertes.fr/hal-0I349264> (consultato il 25 maggio 202I).

Gay F. (2000). Intorno agli omolografi: strumenti e modelli per la geometria descrittiva. Venezia.

Gay F. (2020). A ragion veduta. Immaginazione progettuale, rappresentazione e morfologia degli artefatti. Alghero: Publica.

Grayson C. (a cura di), 1980. Leon Battista Alberti, De pictura. Bari: Laterza.

Kemp M. (1994). La scienza dell'arte. Prospettiva e percezione visiva da Brunelleschi a Seurat. Milano: Giunti.

Migliari R. (2004). Disegno come modello. Riflessioni sul disegno nell'era informatica. Roma: Kappa.

Niceron J.F. (1 646). Thaumaturgus opticus. Paris: Francisci Langlois.

Pavignano M., Cumino C., Zich U. (2020). Catalog Mathematischer Modelle. Connessioni tra testo, rappresentazione grafica e descrizione analitica. In Arena A. et al. (a cura di). Connettere. Un disegno per annodare e tessere. Atti del $42^{\circ}$ Convegno Internazionale dei Docenti delle Discipline della Rappresentazione, pp. 3660-3677. Milano: FrancoAngeli.

Salvatore M. (2020). Prospettici ingegni. Strumenti e metodi per la costruzione della prospettiva applicata. In Disègno n. 6, pp. 95- 108.

Scheiner C. (I63I). Christophori Scheiner, e Societate lesu Germano-Sueui, Pantographice, seu, Ars delineandi res quaslibet per parallelogrammum lineare seu cauum, mechanicum. Roma: Ludouici Grignani.

Seidl E. (20 | 8). Die Modelle, Brill und das studentische Projekt. In Seidl E., Loose F., Bierende E. (Ed.). Mathematik mit Modellen. Alexander von Brill und die Tübinger Modellsammlun, pp. 19-33. Tübingen: Museum der Universität Tübingen MUT.

\section{Autori}

Laura Farroni, Università di RomaTre, laura.farroni@uniroma3.it

Giulia Tarei, Sapienza Università di Roma, giulia.tarei@uniromal .it

Per citare questo capitolo: Farroni Laura, Tarei Giulia (202I). Lo sguardo connettivo: le macchine per disegnare in prospettiva tra XVI e XVII secolo/Connective Eyesight.Tools for Perspective Drawings between XVI e XVII Century. In Arena A Arena M. Mediati D Raffa P (a cura di). Connettere Un disegno per annodare e tessere Linguagoi Distanze Tecnologie. Atti del $42^{\circ}$ Convegno Internazionale dei Docenti delle Discipline della Rappresentazione/Connecting. Drowing for waving retationship. Languges Distances Technologies. Proceedings of the 42 th International Conference of Representation Disciplines Teachers. Milano: FrancoAngeli, pp. 2301 I-2318. 


\title{
Connective Eyesight: Tools for Perspective Drawings between XVI e XVII Century
}

\author{
Laura Farroni \\ Giulia Tarei
}

Abstract

The essay is focused on the way eyesight is the main feature of how drawing machines of the $16^{\text {th }}$ and $17^{\text {th }}$ century evolved. Especially with 'perspectographs' the act of setting one's sight on and to line up one's view relates the beholder with what surrounds him, defining the development of the machines of the Science of Representation themselves. The tool put into relation, each time,'problem and solution', 'real space and space of the representation', 'tri-dimensional and two-dimensional space'. It appears that the mechanism, the automation of the drawing process, connects abstrac-tion and material reality, thus the figurative representations arising from this connection, embed in themselves the cor-respondence among modus operandi and modus cogitandi.

Keywords

drawing machines, perspective, tools, optics, eyesight.

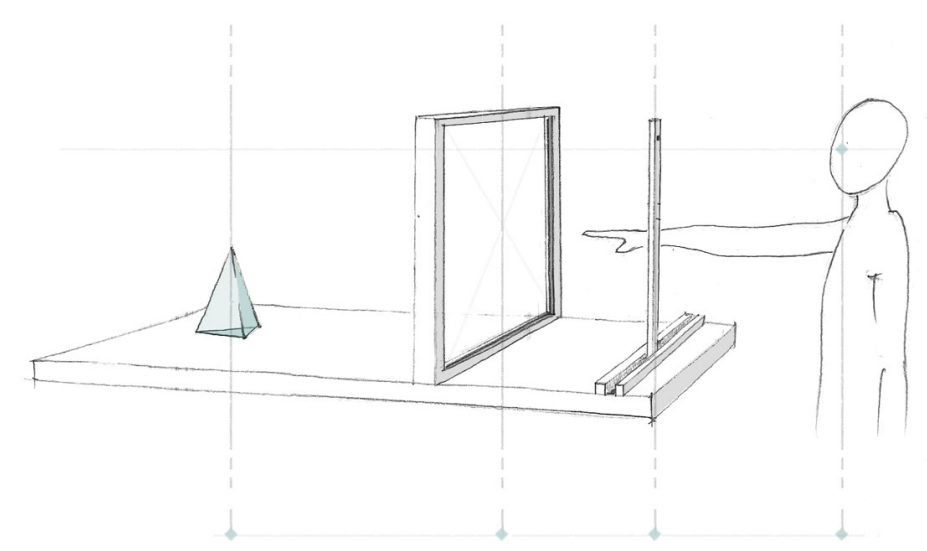




\section{Introduction}

The theme of tools and methods for applied perspective [Salvatore 2020, p. 95] within scientific literature has been the object of numerous studies, where it is scrutinized their employ in project planning, for the carrying over of curves and drawings onto wall surfaces, surveys, as also the aspects relative to a use purely interested in the geometrical interpretation of space, or for didactic reasons.

The explanations of the operating principles of the machines is interpreted through reconstruction of such models, physical and virtual.

There are many collections of machines preserved into museums or universities headquarters: we point out, by way of example, the contribution of the laboratory of mathematical machines of the Modena University.

Many contributions of theoretical character, as well as the experiments of authors such as Fabrizio. Gay, Filippo Camerota, Andrea Casale, Maria G. Bartolini Bussi, Laura Farroni and Marta Salvatore, whose inquiries sometimes present a classificatory approach, a wide vision inclined to systematization, focused sometimes on a particular tool or author, also with respect to the treatises yielded by scholars between the $16^{\text {th }}$ and the $17^{\text {th }}$ centuries.

Recently, many ancient texts were made available through digitalization process and uploaded on the web as open source material.

The essay is focused on the way eyesight is the main feature of how drawing machines of the $16^{\text {th }}$ and $17^{\text {th }}$ century evolved. Especially with 'perspectographs' the act of setting one's sight on and to line up one's view relates the beholder with what surrounds him, defining the development of the machines of the Science of Representation themselves.

During the $\mathrm{XVI}$ and the $\mathrm{XVII}$ centuries, architects, mathematicians, engineers, astronomers employed tools shaping them according to their use.

The protagonists of the century of the Scientific Revolution are figures of scientists that contributed tighter to the development of theories and techniques for the analysis and the codification of the principles regulating the physical world. The work and the researches that those personalities brought forth in the field of the instrumental experimentation for the Science of Representation vouch for the cornerstone aspect of the intrinsic operating mode of this Science: the union of knowing and know-how.

The machine places itself as an element of connection between problem and solution, between real space and the space of representation, between two-dimensional and three-dimensional space. Beginning with the study of the way to set one sight's on in order to depict reality, it is understood how the machines were made and why they differed from each other. The results were collected in an abacus in which two aspects of the machines were considered: the parts and the mode of employ. The parts of the machines do materialize the theorical principles; their assembly guarantees that the geometrical principles are followed and the mechanism returns the perspective representation.

In them we can recognize the beholder, the line of vision, the picture plane, the procedures of projection and cross section. The mode of employ of each tool bears meaning of their descriptive nature.
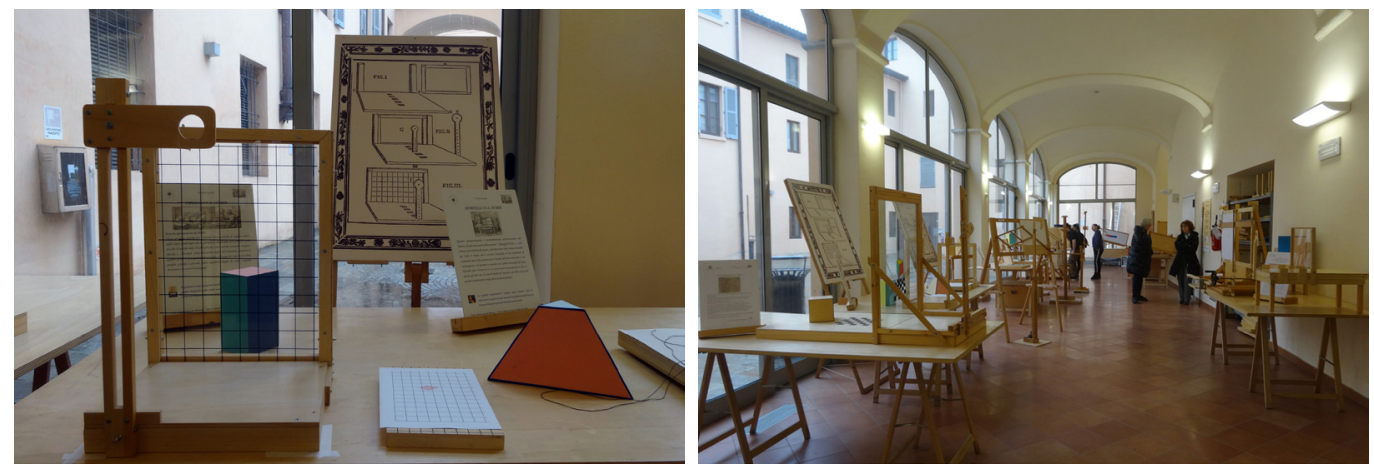
Fig. 3. Albrecht Dürer Glass [Dürer 1525, p.182].

Fig. 4. Albrecht Dürer Window [Dürer 1525 p. 183].
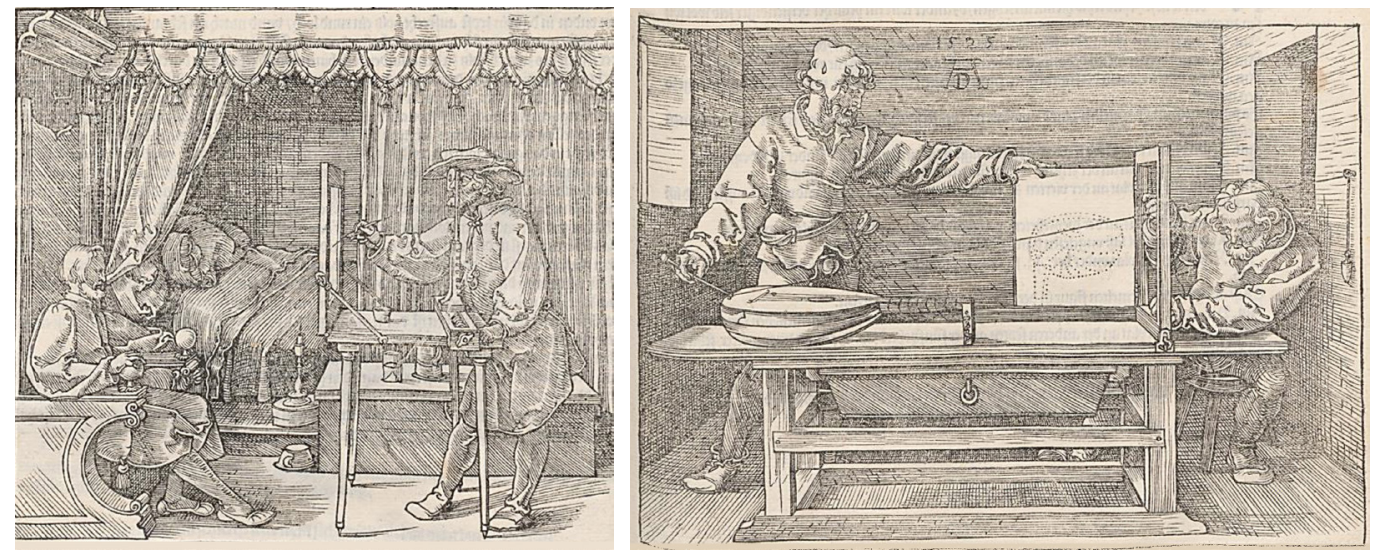

As a premise to recognize and classify the modes of use it must be understood that perspective instruments initially directly comprise the definition of painting given by Alberti in the De Pictura, as 'intersection of the visual pyramid'.

"Quae res cum ita sit, pictam superficiem intuentes intercisionem quandam pyramidis videre videntur. Erit ergo pictura intercisio pyramidis visivae secundum datum intervallum posito centro statutisque luminibus in datam superficiem lineis et coloribus arte repraesentata" [Alberti, I 435, p. 7].

The base of the pyramid is described as a window (the glass), on which for translucence the image of the object is depicted (the 'worn' object) and that incorporates in a direct way the idea of 'intersegazione'.

\section{Definition of the adopted methodologies of research and critical abacus of perspective machines}

The study took advantage of the following methods: a. historical research; $b$. analysis of the geometrical principles and the procedures relative to some machines; $\mathrm{c}$. design and building of some machines; $d$. cataloguing of the outcomes in an abacus.

The research mainly divided itself in three phases: perusal of bibliographic sources; prototypes were realized following these sources; data was collected analyzing the prototypes and the sources in order to fill in the abacus that allows an analytical comparison between the tools of different authors or different tools made by the same author.The machine itself portrays the principles the different authors give shape to when setting the perspective. Those principles are derived from aware choices based on experiments. This aspect highlights the empirical character of the scientific method.

It is observed the fact that perspective machines present a double feature of themselves: a. the ability to acquire metrical, geometrical, and topological data of the physical world, analogous to survey operations; b. the ability to transfer: to obtain the transposition of a two dimensional drawing onto three dimensional space.

The goal of the abacus is to collect systematically the machines for perspective drawing, designed and built in a two centuries span of time and the geometrical principles they group with. The various formal variations deriving either from the progressive overcoming of physical limits, or from the requirements related to specific operations these tools were designed for The machines collected and presented in the abacus are:

- Vetro di Albrecht Dürer XVI century.

- Griglia di Albrecht Dürer XVI century.

- Sportello di Albrecht Dürer XVI century.

- Traguardi di Jacob Keser XVI century.

- Sportello di Albrecht Dürer con diottra (Egnazio. Danti) XVI century. 
Fig. 5. Egnazio Danti, Window with graduated rulers, XVI century [Danti 1583].

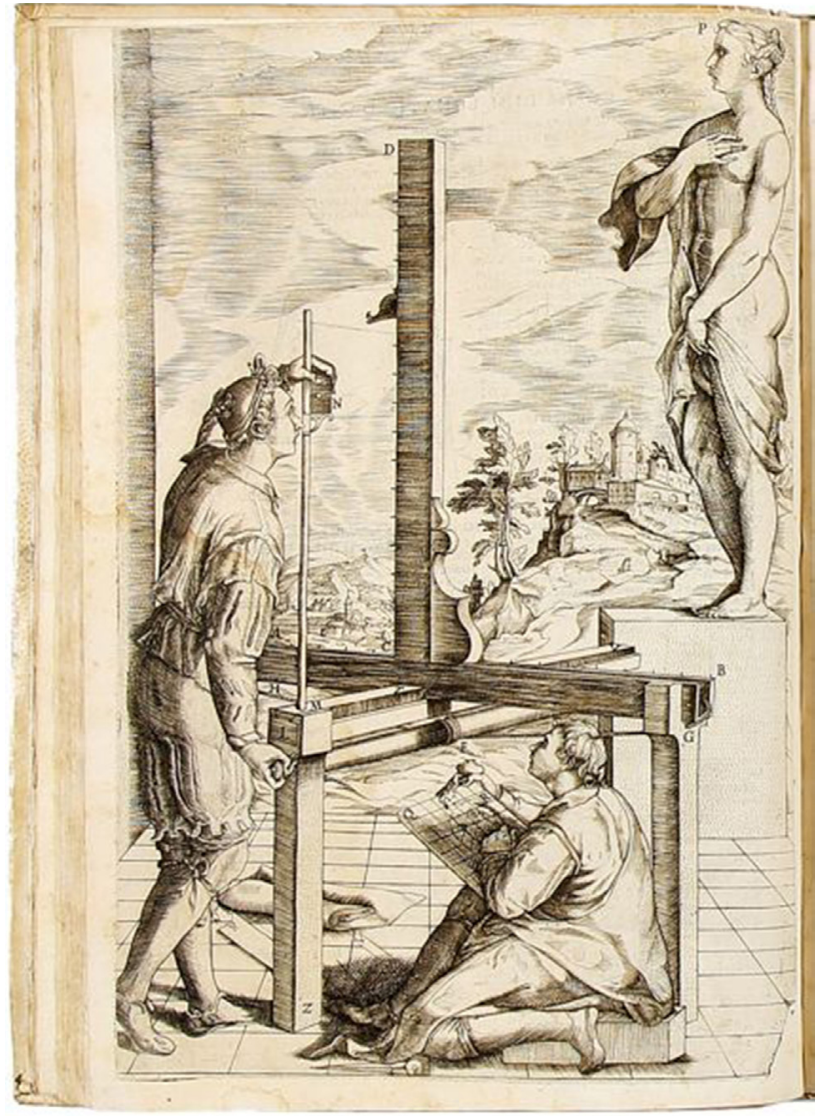

- Prospettografo dell'abate di Lerino (Don Girolamo da Perugia) XVI century.

- Sportello di Egnazio Danti con regoli graduati XVI century.

- Prospettografo di Tigini dè Marij XVI century.

- Prospettografo di lacomo Barozzi da Vignola XVI century.

- Prospettografo di Baldassarre Lanci XVII century.

- Prospettografo di Wenzel Jämnitzer XVII century.

- Prospettografo di Chrostoph Scheiner XVII century.

- Prospettografo di Bettini - Grienberger XVII century.

- Prospettografo di Cigoli - Niçeron XVII century.

Geometrical principles common to all machines define the operative sequence of projection from a single point and intersection on a plane. usually vertically set, or not, given that this plane is perpendicular to the line of view. The lines of sight give back on the plane the points of the representation.

The different designs of the machines depend on these elements, on their being conceived as geometrical elements, and on their translation into physical elements. Those are a point as centre, the visual lines, and the plane of the representation. Designing the machines means to give a shape to these geometrical entities, experimenting time after time the possible matches between tangible and intangible. This way the importance of eyesight stands out.

Different mode of use of the tools are taken into account. The use of the tool depends on the axiomatic system chosen for an effective description.

In order to guarantee this effectiveness, it is necessary to render the projective-descriptive process simple and quick, working on the compactness and practicality of the project of the perspective machine. 
Fig. 6. Prototiype of Chrostoph Scheiner 'perspectograph' XVII sec. made by Marta Pagliaccia for the universitary class of Macchine matematiche. Helded Marof. L. Farroni e P. Marchipe. Dipartimento degli Studi di Roma Tre.
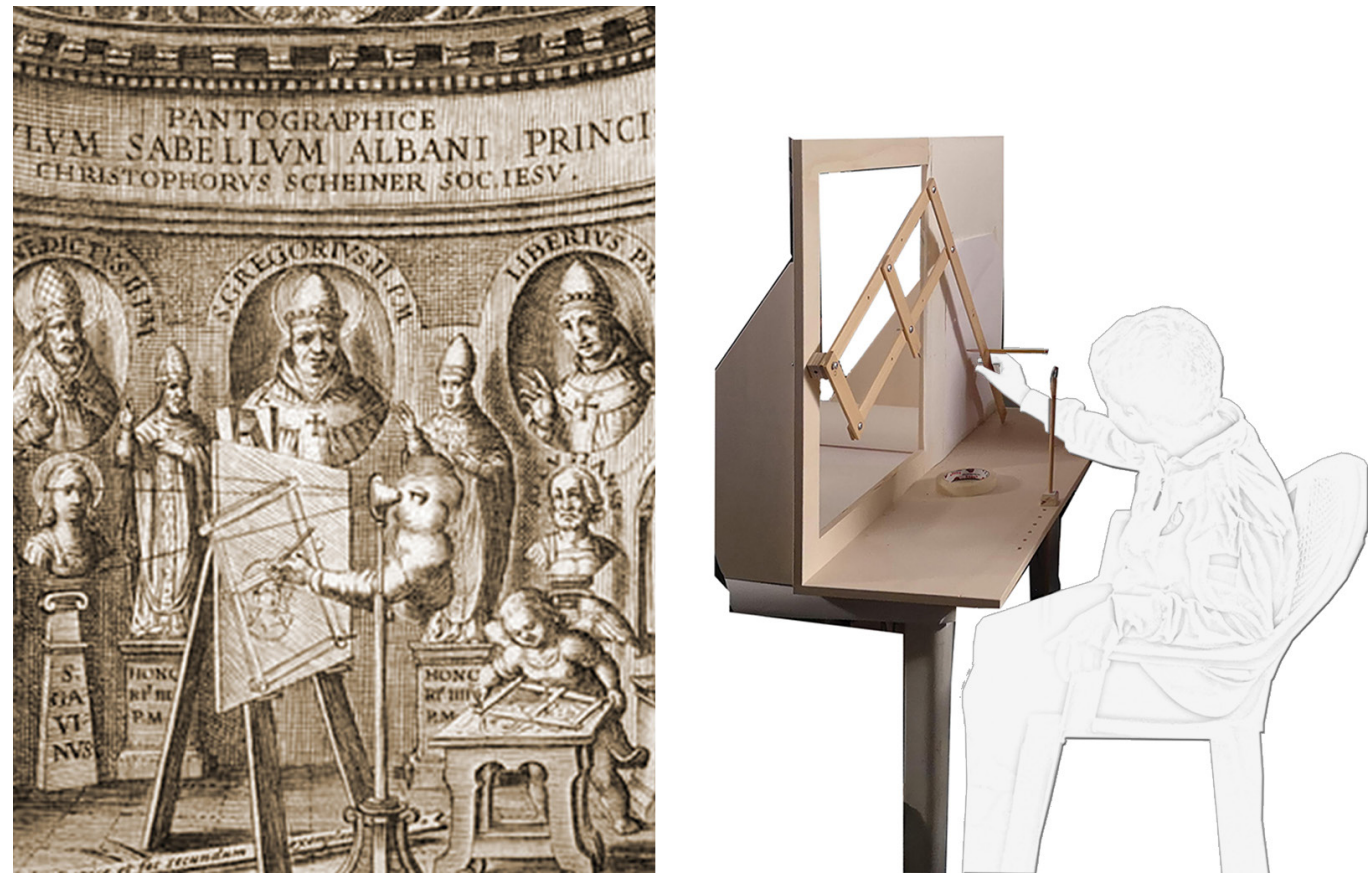

Some solutions expect the designer to stand still in its position of observer, some others -introduction sometimes auxiliary elements- the designer is freed from the position of the actual point of view, which stays unvaried: the designer can move to carry over the drawing, on any surface either integrated to the machine or not, without modifying the perspective's system.

From this is contrived the stratagem of materializing the lines of view by using threads through space or, through what can be defined as a synthesis on the picture plane, with set squares and rulers sliding on it.

All this results in one other operational feature as well: the simultaneousness of the operations of observation and drawing, that can occur in two different phases, spaced out by a suitable measurement.

The method of carrying over from the virtual plan to the plan of the sheet, when it is necessary, asks for, even though implicitly, geometrical transformations, as, for instance, homothethy and translation [Bartolini 2006, p. 39]. The dimensions of the machines, their
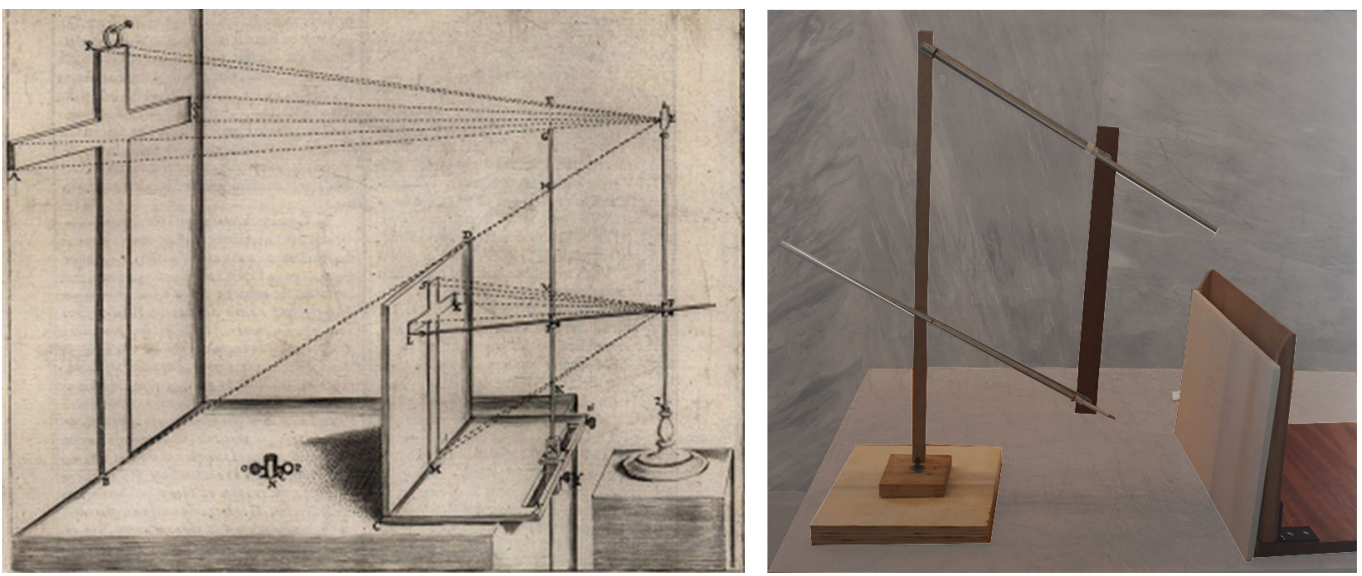

Fig. 7. Prototype of Bettini - Grienberger sec. made by Chiara Egidi for the universitary class of Macchine matematiche. Helded by Prof. L. Farroni e P. Magrone. Dipartimento diArchitettura. Universitò degli Studi di Roma Tre. 
Fig. 8. Prototype of Cigoli - Niçeron 'perspectograph' XVII sec. made by Giulia Tarei for the universitary class of Macchine matematiche. Helded by Prof. L. Farroni e P. Magrone. Dipartimento di Architettura Universit degli Studi di Roma Tre.
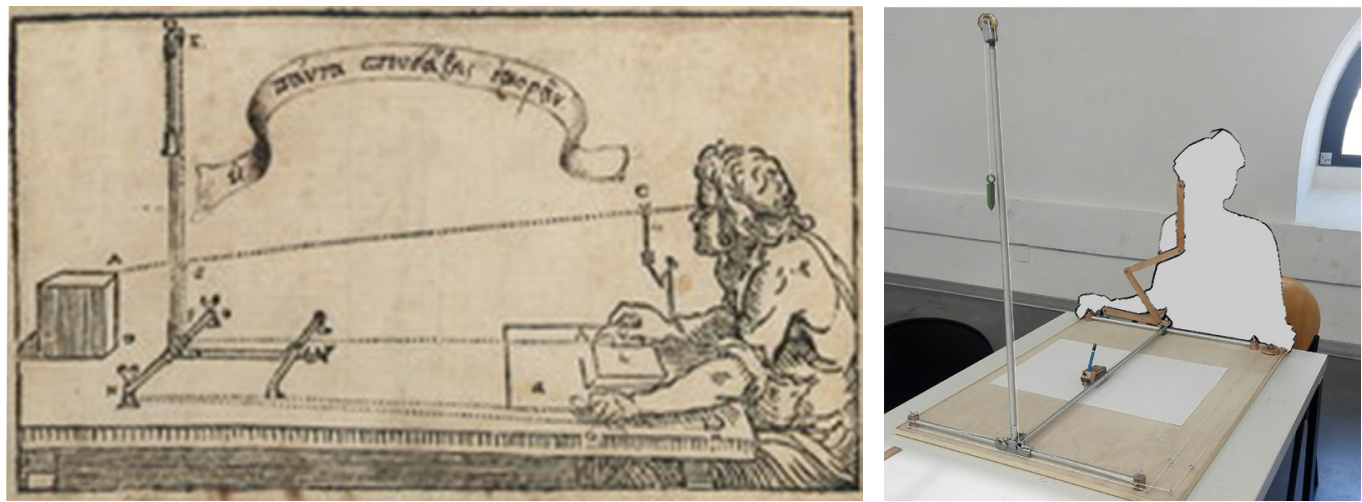

parts and their mutual distances is anthropometric, consenting control over the mode of operation.

With limit we mean the recognition of the measures of the parts composing the machine and of the reciprocal distances between those parts necessary for the machine to work. These limits are operational and dimensional ones depending or being solved by the formal choices.

The human beholder defines the measurement unit and the tool determines the space of the representation. Additional tools support overcoming the anthropometric limits.

\begin{tabular}{|c|c|c|c|c|c|}
\hline & & & XVI secolo & & \\
\hline & $\begin{array}{l}\text { vetro } \\
\text { A.Dürer }\end{array}$ & $\begin{array}{l}\text { griglia } \\
\text { A. Dürer }\end{array}$ & $\begin{array}{l}\text { sportello } \\
\text { A. Dürer }\end{array}$ & $\begin{array}{l}\text { traguardo } \\
\text { J. Keser }\end{array}$ & $\begin{array}{l}\text { sportello } \\
\text { A. Dürer / E. Danti }\end{array}$ \\
\hline osservatore & $\begin{array}{l}\text { conicidente con } \\
\text { punto di vista }\end{array}$ & $\begin{array}{l}\text { conicidente con } \\
\text { punto di vista }\end{array}$ & $\begin{array}{l}\text { non coincidente } \\
\text { con punto di vista }\end{array}$ & $\begin{array}{l}\text { non coincidente } \\
\text { con punto di vista }\end{array}$ & $\begin{array}{c}\text { conicidente con punto } \\
\text { di vista }\end{array}$ \\
\hline punto di vista & oculare & oculare & chiodo su parete & chiodo su parete & diottra \\
\hline piano di quadro & $\begin{array}{l}\text { telaio verticale } \\
\text { con vetro }\end{array}$ & $\begin{array}{l}\text { telaio verticale } \\
\text { con griglia }\end{array}$ & $\begin{array}{l}\text { telaio verticale } \\
\text { con fili diagonali }\end{array}$ & $\begin{array}{l}\text { telaio verticale } \\
\text { con vetro }\end{array}$ & $\begin{array}{l}\text { telaio verticale con fili } \\
\text { diagonali e sportello } \\
\text { con foglio da disegno }\end{array}$ \\
\hline piano orizzontale & piano di supporto & $\begin{array}{l}\text { foglio da disegno } \\
\text { con griglia }\end{array}$ & piano di supporto & piano di supporto & piano di supporto \\
\hline raggio visivo & immateriale & immateriale & filo & filo & filo \\
\hline $\begin{array}{l}\text { elemento } \\
\text { ausiliario }\end{array}$ & - & - & $\begin{array}{l}\text { sportello con } \\
\text { foglio da disegno }\end{array}$ & regolo - visore & $\begin{array}{l}\text { diottra } \\
\text { sportello }\end{array}$ \\
\hline disegnatore & osservatore & osservatore & osservatore & $\begin{array}{l}\text { non coincidente } \\
\text { con l'osservatore }\end{array}$ & osservatore \\
\hline operatori & 1 & 1 & 2 & 2 & 2 \\
\hline scopo & rilievo & rilievo & rilievo & rilievo & rilievo \\
\hline modo d'uso & diretto & indiretto & indiretto & diretto & indiretto \\
\hline operazioni & ricalco sul vetro & $\begin{array}{l}\text { 1.individuazione } \\
\text { di punti su griglia } \\
\text { verticale } \\
\text { 2.riporto su foglio } \\
\text { con griglia }\end{array}$ & $\begin{array}{l}\text { 1.individuazione } \\
\text { di punti su telaio } \\
\text { con diagonali } \\
\text { 2.riporto su } \\
\text { sportello con } \\
\text { foglio }\end{array}$ & disegno sul vetro & $\begin{array}{l}\text { 1.individuazione di } \\
\text { punti su telaio con } \\
\text { diagonali } 2 \text {.riporto } \\
\text { su sportello con foglio }\end{array}$ \\
\hline $\begin{array}{l}\text { limite } \\
\text { antropometrico }\end{array}$ & $\begin{array}{l}\text { lunghezza del } \\
\text { braccio }\end{array}$ & - & - & - & - \\
\hline limite operativo & $\begin{array}{l}\text { disegnatore/ } \\
\text { osservatore in } \\
\text { posizione } \\
\text { vincolata }\end{array}$ & $\begin{array}{l}\text { disegnatore/ } \\
\text { osservatore in } \\
\text { posizione } \\
\text { vincolata }\end{array}$ & $\begin{array}{l}\text { necessità di un } \\
\text { assistente e di } \\
\text { una parete }\end{array}$ & $\begin{array}{l}\text { necessità di un } \\
\text { assistente e di } \\
\text { una parete }\end{array}$ & $\begin{array}{l}\text { necessità di un } \\
\text { assistente }\end{array}$ \\
\hline $\begin{array}{l}\text { limite di } \\
\text { precisione }\end{array}$ & - & $\begin{array}{l}\text { errori dovuti al } \\
\text { riporto su griglia }\end{array}$ & $\begin{array}{l}\text { errori dovuti allo } \\
\text { spostamento del } \\
\text { filo }\end{array}$ & - & $\begin{array}{l}\text { errori dovuti allo } \\
\text { spostamento del filo }\end{array}$ \\
\hline
\end{tabular}




\begin{tabular}{|c|c|c|c|c|c|}
\hline & \multicolumn{5}{|c|}{ XVI secolo } \\
\hline & $\begin{array}{l}\text { prospettografo } \\
\text { Abate da Lerino }\end{array}$ & $\begin{array}{c}\text { prospettografo } \\
\text { E. Danti }\end{array}$ & $\begin{array}{c}\text { prospettografo } \\
\text { O. Trigini De' Marij }\end{array}$ & $\begin{array}{l}\text { prospettografo } \\
\text { I. Barozzi da Vignola }\end{array}$ & $\begin{array}{c}\text { prospettografo } \\
\text { B. Lanci }\end{array}$ \\
\hline osservatore & $\begin{array}{l}\text { conicidente con } \\
\text { punto di vista }\end{array}$ & $\begin{array}{l}\text { conicidente con } \\
\text { punto di vista }\end{array}$ & $\begin{array}{l}\text { conicidente con } \\
\text { punto di vista }\end{array}$ & $\begin{array}{l}\text { conicidente con } \\
\text { punto di vista }\end{array}$ & $\begin{array}{l}\text { conicidente con punto } \\
\text { di vista }\end{array}$ \\
\hline punto di vista & oculare & oculare & osservatore & oculare & oculare \\
\hline piano di quadro & $\begin{array}{l}\text { telaio verticale e } \\
\text { sportello con foglio } \\
\text { da disegno }\end{array}$ & $\begin{array}{l}\text { sistema di aste } \\
\text { graduate } \\
\perp \text { tra di loro }\end{array}$ & $\begin{array}{l}\text { telaio per } \\
\text { osservare }\end{array}$ & $\begin{array}{l}\text { sistema di aste } \\
\text { graduate } \\
\perp \text { tra di loro }\end{array}$ & piano cilindrico \\
\hline piano orizzontale & piano di supporto & foglio da disegno & - & asta orizzontale & piano di supporto \\
\hline raggio visivo & immateriale & immateriale & immateriale & immateriale & immateriale \\
\hline $\begin{array}{l}\text { elemento } \\
\text { ausiliario }\end{array}$ & $\begin{array}{l}\text { squadre - righelli } \\
\text { su piano di quadro }\end{array}$ & $\begin{array}{l}\text { due regoli graduati } \\
\perp \text { tra di loro }\end{array}$ & $\begin{array}{l}\text { - foglio da disegno } \\
\text { contiguo al telaio } \\
\text { - filo }\end{array}$ & $\begin{array}{c}\text { regoli } \\
\text { foglio con griglia }\end{array}$ & puntatore \\
\hline disegnatore & osservatore & osservatore & osservatore & assistente & osservatore, puntatore \\
\hline operatori & 1 & 1 & 1 & 2 & 1 \\
\hline scopo & rilievo & rilievo e riporto & rilievo & rilievo e riporto & rilievo e riporto \\
\hline modo d'uso & indiretto & indiretto & diretto & indiretto & diretto \\
\hline operazioni & $\begin{array}{l}\text { 1.individuazione di } \\
\text { punti su telaio con } \\
\text { righelli 2.riporto su } \\
\text { sportello con foglio }\end{array}$ & $\begin{array}{l}\text { 1.individuazione } \\
\text { di punti con regolo } \\
\text { verticale } \\
\text { 2.riporto su foglio } \\
\text { con regolo } \\
\text { orizzontale }\end{array}$ & $\begin{array}{l}\text { osservazione su } \\
\text { quadro virtuale e } \\
\text { riporto su quadro } \\
\text { reale prendendo le } \\
\text { misure su un } \\
\text { regolo }\end{array}$ & $\begin{array}{l}\text { 1.individuazione } \\
\text { di punti su aste } \\
\text { graduate } \\
\text { 2.riporto di misure } \\
\text { su foglio con } \\
\text { griglia }\end{array}$ & $\begin{array}{l}\text { operazioni simultanee } \\
\text { di osservazione e } \\
\text { disegno }\end{array}$ \\
\hline $\begin{array}{l}\text { limite } \\
\text { antropometrico }\end{array}$ & - & - & - & - & - \\
\hline limite operativo & - & - & - & $\begin{array}{l}\text { - complessità } \\
\text { dello strumento - } \\
\text { necessità di un } \\
\text { assistente }\end{array}$ & $\begin{array}{l}\text { lo strumento consente } \\
\text { di riportare un disegno } \\
\text { in prospettiva su } \\
\text { parete curva }\end{array}$ \\
\hline $\begin{array}{l}\text { limite di } \\
\text { precisione }\end{array}$ & - & - & - & - & - \\
\hline
\end{tabular}

\section{Conclusions}

Starting this investigation, the characteristics of the machines were analysed and compared. This is a critical evolutionary framework of the technological process.

The tools work as geometrical model, and are direct expression of a certain aspect or phenomenon giving tangible shape to an abstract theory [Seidl 20।8, p.20].

Studying the bibliographical sources, and based on the descriptions of the authors, prototypes were realized. Relating together the elements of the investigation, the geometrical principles and common aspects were put into focus, and the data that structures the abacus collected. The paper shed light onto logical, chronological, and personal links among scientists. In the comparison between the analysed prototypes, the employ of threads to simulate the line of view, groups, for instance, Dürer's device with the one by Keser, and with the tool by Jämnitzer realized a century later. The different attempts to separate the person who draws from the beholder's position -especially for- has as outcome the need for two operators, as for instance in the late experiments of Dürer, in those by Danti, Keser and of Vignola, before achieving what the automations by Bettini and Cigoli allowed.

The mechanism, the process automation connects the superior level of abstraction with the material world. The figurative representation deriving from this nexus embodies the way that the modus operandi and the modus cogitandi mirror each other. 


\begin{tabular}{|c|c|c|c|c|}
\hline & \multicolumn{4}{|c|}{ XVII secolo } \\
\hline & $\begin{array}{c}\text { prospettografo } \\
\text { W. Jämnitzer }\end{array}$ & $\begin{array}{c}\text { prospettografo } \\
\text { C. Scheiner }\end{array}$ & $\begin{array}{l}\text { prospettografo } \\
\text { Bettini - Grienberger }\end{array}$ & $\begin{array}{l}\text { prospettografo } \\
\text { L.Cigoli - J.F. Niceron }\end{array}$ \\
\hline osservatore & $\begin{array}{l}\text { conicidente con } \\
\text { punto di vista }\end{array}$ & conicidente con punto di vista & $\begin{array}{l}\text { conicidente con } \\
\text { punto di vista }\end{array}$ & $\begin{array}{l}\text { conicidente con punto } \\
\text { di vista }\end{array}$ \\
\hline punto di vista & oculare & oculare & oculare & oculare \\
\hline piano di quadro & $\begin{array}{l}\text { sistema di aste } \\
\perp \text { tra di loro }\end{array}$ & telaio per osservare & foglio verticale & $\begin{array}{l}\text { sistema di aste } \\
\text { mobili } \perp \text { tra di loro }\end{array}$ \\
\hline piano orizzontale & foglio da disegno & - & - & $\begin{array}{l}\text { piano di supporto con } \\
\text { foglio da disegno }\end{array}$ \\
\hline raggio visivo & filo & immateriale & $\begin{array}{l}\text { asta orizzontale } \\
\text { (radius visualis) }\end{array}$ & immateriale \\
\hline $\begin{array}{l}\text { elemento } \\
\text { ausiliario }\end{array}$ & $\begin{array}{l}\text { asta verticale } \\
\text { graduata } \\
\text { pianta e alzato }\end{array}$ & $\begin{array}{l}\text { - foglio da disegno contiguo } \\
\text { al telaio } \\
\text { - parallelogramma articolato } \\
\text { - puntatore e tracciatore }\end{array}$ & $\begin{array}{l}\text { asta orizzontale } \\
\text { (radius scriptorius) } \\
\text { perno per far ruotare } \\
\text { l'asta verticale }\end{array}$ & carrucole e cursore \\
\hline disegnatore & osservatore & osservatore & osservatore & osservatore \\
\hline operatori & 1 & 1 & 1 & 1 \\
\hline scopo & rilievo e riporto & rilievo & rilievo e riporto & rilievo e riporto \\
\hline modo d'uso & indiretto & diretto & diretto & diretto \\
\hline operazioni & $\begin{array}{l}\text { 1.individuazione } \\
\text { di punti in pianta } \\
\text { e alzato } \\
\text { 2.riporto di quote } \\
\text { su asta verticale } \\
\text { 3.ribaltamento } \\
\text { asta e misure su } \\
\text { piano orizzontale }\end{array}$ & $\begin{array}{l}\text { - operazioni simultanee di } \\
\text { osservazione e disegno } \\
\text { - lo strumento sfrutta la } \\
\text { trasformazione geometrica } \\
\text { omotetica } \\
\text { - automatismo }\end{array}$ & $\begin{array}{l}\text { - operazioni } \\
\text { simultanee di } \\
\text { osservazione e } \\
\text { disegno } \\
\text { - lo strumento sfrutta } \\
\text { la trasformazione } \\
\text { geometrica di } \\
\text { traslazione } \\
\text { - automatismo }\end{array}$ & $\begin{array}{l}\text { - operazioni } \\
\text { simultanee di } \\
\text { osservazione e } \\
\text { disegno } \\
\text { - automatismo }\end{array}$ \\
\hline $\begin{array}{l}\text { limite } \\
\text { antropometrico }\end{array}$ & - & - & - & - \\
\hline limite operativo & $\begin{array}{l}\text { lo strumento } \\
\text { prevede l'utilizzo } \\
\text { di disegni ausiliari } \\
\text { pianta e alzato }\end{array}$ & - & - & - \\
\hline $\begin{array}{l}\text { limite di } \\
\text { precisione }\end{array}$ & - & - & - & - \\
\hline
\end{tabular}

The machine is in itself the choice of one axiomatic system and implies the geometrical knowledge and the projective skills. This choice determines the variety of the technical solutions for perspective drawing, too. The solutions presented for the projection of physical points of an object for the intersection between visual lines and the plane are materialized in several ways.

Writing this abacus the objective is to define the evolution of the technical skills and knowledge in relation to the theoretical developments. The authors also wanted to highlight the managing of meaningful criteria leading to a parametric method, in view of possible future transfer to virtual applications. For this reason the path of research is not set once and for all. Every choice reciprocates one particular type of drawing, and the geometrical control.

Each machine and the principle within it is the product of an aware choice based on the state of the science.

All machines are actually a selected synthesis of theoretical principles.

References

Barbaro D. (I568). La pratica della perspettiva ... Opera molto vtile a pittori, a scultori, \& ad architetti. Venezia: Camillo, \& Rutilio Borgominieri fratelli al segno di S. Giorgio.

Bartoli M.T., Lusoli M. (20 I5). Le teorie, le tecniche, i repertori figurativi nella prospettiva d'architettura tra il '400 e il '700. Firenze: University Press. 
Bartolini Bussi M.G., Maschietto M. (2006). Macchine matematiche: dalla storia alla scuola.Verlag: Springer.

Bettini M. (1645). Apiaria Universae Philosophiae Mathematicae. Bologna: JO. Bapt. Ferronius - Bononiae.

Camerota F. (2003). II distanziometro di Baldassarre Lanci: prospettiva e cartografia militare alla corte dei Medici. In Galluzzi P., Beretta M.,Triarico C. (a cura di). Musa Musaei. Studies on Scientific Instruments and Collections in Honour of Mara Miniati. Firenze: Leo S. Olschki Editore, pp. 79-92.

Camerota F. (20 I0). Arte e Scienza da Leonardo a Galileo. Firenze: Giunti.

Camerota F. (20 I 0). Linear Perspective in the Age of Galileo. Ludovico Cigoli's Prospettiva pratica. Firenze: Leo S. Olschki Editore.

Casale A. (20।8). Forme della percezione. Dal pensiero all'immagine. Milano: FrancoAngeli.

Cigoli Cardi L. (16|3). II Cigoli. Prospettiva pratica di Fra Lodovico Cardi Cigoli Cavaliere della Sacra et Illustre Religione di San Giovanni Hierosolomitasano, dimostrata con tre regole e la descrizione di due strumenti da tirare in prospettiva e modo di adoperarli, et cinque ordini di architettura con le loro misure. Firenze: Gabinetto Disegni e Stampe degli Uffizi.

Danti E. (I583). Le due regole della prospetiva pratica di M.lacomo Barozzi da Vignola. con commentarii del reverendo Padre Maestro Egnatio Danti dell'ordine dei predicatori matematico dello studio di Bologna. Roma: Francesco Zannetti.

Dürer A. ( I 525). Underweysung der Messung mit dem Zyrckel und Richtscheyt. Nurnberg: Hieronymus Andreae.

Farroni L., Magrone P. (20 I 8). A Hands-on Laboratory with Mathematical Mechanical Drawing Machines. In Conference Proceedings, Bridges 2018 Mathematics, Art, Music, Architecture, Education, Culture. Stoccolma 25-27 luglio 20 I8, pp. 617-622.

Farroni L., Magrone P. (2019). A Multidisciplinary Approach to Teaching Mathematics and Architectural Representation: Historical Drawing Machines. Relations between Mathematics and Drawing. In History and Pedagogy of Mathematics, July 20 I6, Montpellier, France. <https://hal.archives-ouvertes.fr/hal-0I349264> (accessed 202I, May 25).

Gay F. (2000). Intorno agli omolografi: strumenti e modelli per la geometria descrittiva.Venezia.

Gay F. (2020). A ragion veduta. Immaginazione progettuale, rappresentazione e morfologia degli artefatti. Alghero: Publica.

Grayson C. (a cura di), 1980. Leon Battista Alberti, De pictura. Bari: Laterza.

Kemp M. (1994). La scienza dell'arte. Prospettiva e percezione visiva da Brunelleschi a Seurat. Milano: Giunti.

Migliari R. (2004). Disegno come modello. Riflessioni sul disegno nell'era informatica. Roma: Kappa.

Niceron J.F. ( 1646$)$. Thaumaturgus opticus. Paris: Francisci Langlois.

Pavignano M., Cumino C., Zich U. (2020). Catalog Mathematischer Modelle. Connessioni tra testo, rappresentazione grafica e descrizione analitica. In Arena A. et al. (a cura di). Connettere. Un disegno per annodare e tessere. Atti del $42^{\circ}$ Convegno Internazionale dei Docenti delle Discipline della Rappresentazione, pp. 3660-3677. Milano: FrancoAngeli.

Salvatore M. (2020). Prospettici ingegni. Strumenti e metodi per la costruzione della prospettiva applicata. In Disègno n. 6, pp. 95- 108 .

Scheiner C. (1631). Christophori Scheiner, e Societate lesu Germano-Sueui, Pantographice, seu, Ars delineandi res quaslibet per parallelogrammum lineare seu cauum, mechanicum. Roma: Ludouici Grignani.

Seidl E. (20 I8). Die Modelle, Brill und das studentische Projekt. In Seidl E., Loose F., Bierende E. (Ed.). Mathematik mit Modellen. Alexander von Brill und die Tübinger Modellsammlun, pp. 19-33. Tübingen: Museum der Universität Tübingen MUT.

\section{Authors}

Laura Farroni, Università di Roma Tre, laura.farroni@uniroma3.it

Giulia Tarei, Sapienza Università di Roma, giulia.tarei@uniromal .it

To cite this chapter. Farroni Laura, Tarei Giulia (202I). Lo sguardo connettivo: le macchine per disegnare in prospettiva tra XVI e XVII secolo/Connective Eyesight:Tools for Perspective Drawings between XVI e XVII Century. In Arena A. Arena M. Mediati D., Raffa P. (a cura di). Connettere Un disegno per annodare e tessere Linguaggi Distanze Tecnologie. Atti del $42^{\circ}$ Convegno Internazionale dei Docenti delle Discipline della Rappresentazionel Connecting Drowing for wo Disciplines Teachers. Milano: FrancoAngeli, pp. 2301-2318. 